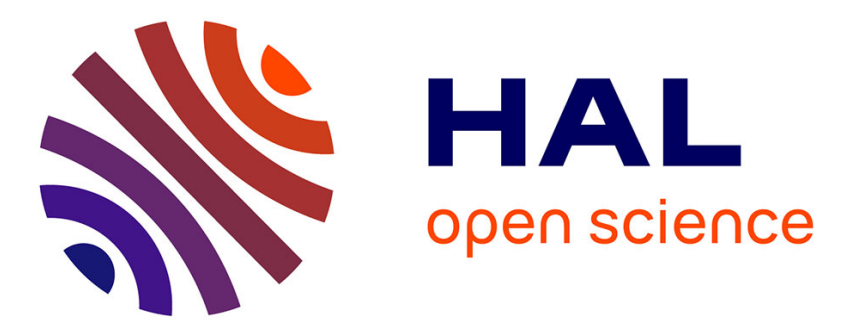

\title{
Large Magneto-ionic Variations toward the Galactic Center Magnetar, PSR J1745-2900
}

Gregory Desvignes, Ralph Eatough, Ue-Li Pen, Kejia Lee, S.A. Mao, R. Karuppusamy, Dominic Schnitzeler, Heino Falcke, Michael Kramer, O. Wucknitz, et al.

\section{To cite this version:}

Gregory Desvignes, Ralph Eatough, Ue-Li Pen, Kejia Lee, S.A. Mao, et al.. Large Magneto-ionic Variations toward the Galactic Center Magnetar, PSR J1745-2900. Astrophys.J.Lett., 2018, 852 (1), pp.L12. 10.3847/2041-8213/aaa2f8 . hal-01703746

\section{HAL Id: hal-01703746 \\ https://hal.science/hal-01703746}

Submitted on 10 Dec 2019

HAL is a multi-disciplinary open access archive for the deposit and dissemination of scientific research documents, whether they are published or not. The documents may come from teaching and research institutions in France or abroad, or from public or private research centers.
L'archive ouverte pluridisciplinaire $\mathbf{H A L}$, est destinée au dépôt et à la diffusion de documents scientifiques de niveau recherche, publiés ou non, émanant des établissements d'enseignement et de recherche français ou étrangers, des laboratoires publics ou privés. 


\title{
Large Magneto-ionic Variations toward the Galactic Center Magnetar, PSR J1745-2900
}

\author{
G. Desvignes ${ }^{1}$ (1), R. P. Eatough ${ }^{1}$, U. L. Pen ${ }^{2,3,4,5}$, K. J. Lee ${ }^{6}$, S. A. Mao ${ }^{1}$ (1),

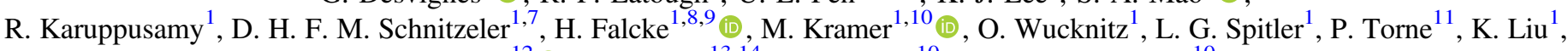 \\ G. C. Bower ${ }^{12}$ (D) I. Cognard ${ }^{13,14}$, A. G. Lyne ${ }^{10}$, and B. W. Stappers ${ }^{10}$ \\ ${ }^{1}$ Max-Planck-Institut für Radioastronomie, Auf dem Hügel 69, D-53121 Bonn, Germany; gdesvignes@mpifr-bonn.mpg.de, reatough@mpifr-bonn.mpg.de \\ ${ }^{2}$ Canadian Institute for Theoretical Astrophysics, University of Toronto, 60 St. George Street, Toronto, Ontario M5S 3H8, Canada \\ ${ }^{3}$ Dunlap Institute for Astronomy and Astrophysics, University of Toronto, 50 St. George Street, Toronto, Ontario M5S 3H4, Canada \\ ${ }^{4}$ Canadian Institute for Advanced Research, CIFAR Program in Gravitation and Cosmology, Toronto, Ontario M5G 1Z8, Canada \\ ${ }^{5}$ Perimeter Institute for Theoretical Physics, 31 Caroline Street North, Waterloo, Ontario, N2L 2Y5, Canada \\ ${ }^{6}$ Kavli institute for astronomy and astrophysics, Peking University, Beijing 100871, People's Republic of China \\ ${ }^{7}$ Bendenweg 51, D-53121 Bonn, Germany \\ ${ }^{8}$ Department of Astrophysics, Institute for Mathematics, Astrophysics and Particle Physics, Radboud University, P.O. Box 9010, \\ 6500 GL Nijmegen, The Netherlands \\ ${ }^{9}$ ASTRON, P.O. Box 2, 7990 AA Dwingeloo, The Netherlands \\ ${ }^{10}$ Jodrell Bank Centre for Astrophysics, School of Physics and Astronomy, The University of Manchester, Manchester M13 9PL, UK \\ ${ }^{11}$ Instituto de Radioastronomía Milimetrica, Avda. Divina Pastora 7, Núcleo Central, E-18012 Granada, Spain \\ ${ }^{12}$ Academia Sinica Institute of Astronomy and Astrophysics, 645 N. A'ohoku Place, Hilo, HI 96720, USA \\ ${ }^{13}$ Laboratoire de Physique et Chimie de l'Environnement et de l'Espace LPC2E CNRS-Université d'Orléans, F-45071 Orléans, France \\ ${ }^{14}$ Station de radioastronomie de Nançay, Observatoire de Paris, CNRS/INSU F-18330 Nançay, France \\ Received 2017 November 28; revised 2017 December 18; accepted 2017 December 19; published 2018 January 3
}

\begin{abstract}
Polarized radio emission from PSR J1745-2900 has already been used to investigate the strength of the magnetic field in the Galactic center (GC), close to Sagittarius A*. Here we report how persistent radio emission from this magnetar, for over four years since its discovery, has revealed large changes in the observed Faraday rotation measure (RM), by up to $3500 \mathrm{rad} \mathrm{m}^{-2}$ (a $5 \%$ fractional change). From simultaneous analysis of the dispersion measure, we determine that these fluctuations are dominated by variations in either the projected magnetic field or the free electron content within the GC, along the changing line of sight to the rapidly moving magnetar. From a structure function analysis of RM variations, and a recent epoch of rapid change of RM, we determine a minimum scale of magneto-ionic fluctuations of size $\sim 2$ au at the GC distance, inferring PSR J1745-2900 is just $\sim 0.1 \mathrm{pc}$ behind an additional scattering screen.
\end{abstract}

Key words: Galaxy: center - magnetic fields - pulsars: individual (J1745-2900)

\section{Introduction}

Measurements of Faraday Rotation in the polarized emission of radio sources can be used to examine the strength and structure of the magnetic field in the interstellar medium (Beck \& Wielebinski 2013). A recent notable example is the radio-loud magnetar, PSR J1745-2900, which displays a high rotation measure $(\mathrm{RM})$ of $-66960 \pm 50 \mathrm{rad} \mathrm{m}^{-2}$ as measured in 2013 May, second only in the Galaxy to the $\mathrm{RM}=$ $(-4.3 \pm 0.1) \times 10^{5} \mathrm{rad} \mathrm{m}^{-2}$ of the supermassive black hole candidate, Sagittarius $A^{*}\left(\operatorname{Sgr} A^{*}\right)$, caused predominantly by the accretion flow on scales smaller than the Bondi-Hoyle radius (Bower et al. 2003). This magnetar therefore allowed first-order estimates of the strength of the magnetic field at the beginnings of the Bondi-Hoyle accretion flow of Sgr A*; $\sim 8 \mathrm{mG}$ at scales of $\sim 0.1$ pc (Eatough et al. 2013). The magnitude and spatial or time variability of the magnetic field also allows models of the accretion flow to be investigated (Pang et al. 2011).

PSR J1745-2900 has also been used to examine the scattering of radio waves toward the Galactic center (GC) by measurements of both the temporal pulse broadening (Spitler et al. 2014) and the angular image broadening (Bower et al. 2014). Combination of the two measurements indicates the principal scattering screen toward the GC is $5.8 \pm 0.3 \mathrm{kpc}$ in front of the magnetar (Bower et al. 2014).

Atypically for magnetars, PSR J1745-2900 has remained active in the radio band for over four years since its discovery at X-ray wavelengths in 2013 (Kennea et al. 2013; Mori et al. 2013).
This has allowed repeated measurements of the RM and dispersion measure (DM). Because the magnetar has a total proper motion of $6.37 \pm 0.16$ mas yr $^{-1}$ relative to Sgr A* (Bower et al. 2015), the time-variations in the measurements of DM and $\mathrm{RM}$ presented here occur along different sightlines. The physical scales probed in the GC are therefore directly related to the observing cadence, and the transverse velocity of the magnetar.

In this paper, long-term polarimetric observations of PSR J1745-2900 with the Effelsberg and Nançay radio telescopes are presented. Section 2 gives a description of the observational campaign and data analysis techniques used. In Section 3, the results of the analysis are presented, and in Section 4 we turn to physical interpretations of the results.

\section{Observations}

Following the discovery of radio pulsations from PSR J1745 -2900 in 2013 April, this source has been monitored with three European radio telescopes operating at complementary observing frequencies. These are the Effelsberg radio telescope, the Nançay Radio Telescope (NRT) and the Jodrell Bank, Lovell radio telescope. In this work, we only refer to measurements from the Effelsberg telescope and the NRT because observations with the Lovell telescope, at a lower frequency of $1.4 \mathrm{GHz}$, suffer from instrumental depolarization due to the large RM. 


\subsection{Effelsberg}

PSR J1745-2900 is observed with the Effelsberg telescope typically on a monthly basis, and fortnightly since 2017 January. One-hour observations at central frequencies of 8.35 and $4.85 \mathrm{GHz}$ are recorded with the PSRIX backend (Lazarus et al. 2016).

The $500 \mathrm{MHz}$ bandwidth provided by the PSRIX backend is first digitized and split into 512 or 1024 channels when observing at $8.35 \mathrm{GHz}$ and $4.85 \mathrm{GHz}$, respectively. The channelized data are then coherently dedispersed at a DM of $1778 \mathrm{pc} \mathrm{cm}^{-3}$, the initial DM value measured by Eatough et al. (2013) and finally folded at the period of the magnetar to create "single pulse profiles."

Since 2017 March, the new "C+" broadband receiver has been available for pulsar observations. Two $2 \mathrm{GHz}$ bands (covering 4-8 GHz) are fed into the new PSRIX2 backend, consisting of two CASPER ${ }^{15}$ ROACH2 boards. Each board digitizes the signal and acts as a full-Stokes spectrometer, creating 2048 frequency channels every $8 \mu$ s. The data are later dedispersed and folded to create single pulse profiles. During the commissioning phase of PSRIX2, the $\mathrm{C}+$ observations replace the $8.35 \mathrm{GHz}$ observations.

\subsection{Nançay}

Observations with the NRT were carried out with the NUPPI instrumentation on average every four days between 2013 May and 2014 August, before resuming at a monthly cadence between 2017 January and July. The setup of the observations was already presented in Eatough et al. (2013), Spitler et al. (2014), and Torne et al. (2015). We briefly summarize it here. A bandwidth of $512 \mathrm{MHz}$ centered at $2.5 \mathrm{GHz}$ is split into 1024 channels and coherently dedispersed using an initial DM value of $1840 \mathrm{pc} \mathrm{cm}^{-3}$ (the best DM value derived from timing of the scattered pulse profiles) then folded at the period of the magnetar and written to disk every $30 \mathrm{~s}$.

\subsection{Post-processing and Calibration}

All the data presented here are corrected for the gain and phase difference between the feeds of the various receivers used. This is achieved by standard pulsar calibration techniques that use observations of a polarized pulsed noise diode. Large $|\mathrm{RM}| \mathrm{s}$, in combination with wide frequency channels, lead to instrumental depolarization. Following the analysis presented in Schnitzeler \& Lee (2015), we calculate that an $|\mathrm{RM}|$ of $7 \times 10^{4} \mathrm{rad} \mathrm{m}^{-2}$ depolarizes the signal by only $4 \%$ in the $2.5 \mathrm{GHz}$ band; at the higher observing frequencies, this effect is negligible. The data reduction is achieved with the standard PSRCHIVE package (Hotan et al. 2004).

\section{Results}

Monitoring of the dispersive and polarization properties of PSR J1745-2900 over a period of $\sim 54$ months has revealed a rather constant DM of $1762 \pm 11 \mathrm{~cm} \mathrm{pc}^{-3}$, while variations in $\mathrm{RM}>3500 \mathrm{rad} \mathrm{m}^{-2}$ are observed. Because $\mathrm{RM}$ is proportional to both projected magnetic field strength $B_{/ /}$, and the free electron density $n_{\mathrm{e}}$, along the line of sight $s, \mathrm{RM} \propto \int B_{/ /}(s) n_{\mathrm{e}}(s) d s$, it is important to disentangle these quantities to understand the

\footnotetext{
15 https://casper.berkeley.edu/
}

physical mechanisms causing the variations in RM. For PSR J1745-2900, which has the highest DM among all the known pulsars, the measurement of the DM is influenced by pulse scattering. In this section, the methods used to measure both the DM and RM are described, as well as the results of our monitoring campaign.

\subsection{DM Variations}

To accurately measure the DM and remove the bias caused by the scattering of the pulse profile (and possibly the variations of it), we modeled both scattering and DM simultaneously over a range of frequency subbands for each NUPPI, PSRIX, and PSRIX2 observations. Given the low amount of dispersion across the band of the PSRIX data at $8.35 \mathrm{GHz}(\sim 12 \mathrm{~ms})$, we did not apply this technique to these data.

Following Spitler et al. (2014), we use a scattered Gaussian pulse function to model the single pulses observed between 4 and $8 \mathrm{GHz}$ and the averaged pulse profile at $2.5 \mathrm{GHz}$. However, in contrast to Spitler et al. (2014), we did not correct for the jittering of the single pulses to create an average "de-jittered" profile. Instead, we model simultaneously some of the brightest single pulses in each observation with different Gaussian widths $\sigma$ to increase the significance of our results.

The scattered Gaussian pulse profile function for a single channel is given by Equation (3) of Spitler et al. (2014). We extend it here for multiple channels to include the DM as a parameter. We can therefore write the likelihood $\Lambda$ as

$$
\log \Lambda=-\frac{1}{2} \sum_{i}^{N_{\mathrm{p}}} \sum_{j}^{N_{\mathrm{c}}} \sum_{k}^{N_{\mathrm{b}}}\left\{\frac{\left[P_{i j k}-T_{i j k}\right]^{2}}{\sigma_{i j}^{2}}\right\}+\text { const },
$$

where $P_{i j k}$ is the observed single pulse profile $i$ with frequency channel $j$ and profile bin $k$ over $N_{\mathrm{p}}$ profiles included in the modeling with $N_{\mathrm{c}}$ frequency channels and $N_{\mathrm{b}}$ phase bins. $\sigma_{i j}^{2}$ is the variance of the noise in the profile $i, j . T_{i j k}$ is the modeled scattered profile,

$$
\begin{aligned}
T_{i j k}= & \frac{A_{i j} \sqrt{\pi}}{2} w_{i} e^{\frac{w_{i}^{2}-4 \phi_{i j k} \tau}{4 \tau^{2}}} \\
& \times\left\{1+\operatorname{erf}\left(\frac{2 \tau\left(\phi_{i j k}-\phi_{0, i j}\right)-w_{i}^{2}}{2 \tau w_{i}}\right)\right\}+b_{i j} .
\end{aligned}
$$

$A_{i j}$ and $b_{i j}$ are, respectively, the amplitude and the baseline offset of channel $j$ from profile $i$. $\phi_{0, i j}$ is the phase center of the Gaussian of width $w_{i}$, but delayed in each channel because of dispersion. $\phi_{0, i j}$ is given by $\phi_{0, i j}=\phi_{0, i}+k \mathrm{DM} \times\left(1 / f_{i}^{2}-1 / f_{0}^{2}\right)$, where the dispersion constant $k=4.15 \times 10^{3} \mathrm{MHz}^{2} \mathrm{~cm}^{3} \mathrm{pc}^{-1} \mathrm{~s}$. Finally, $\tau$ is the scattering time at a reference frequency $f_{0}$. The dimensionality of the model is therefore $2+N_{\mathrm{p}} \times\left(2+2 \times N_{\mathrm{c}}\right)$, where $N_{\mathrm{p}}$ is 1 for the NRT averaged profile, typically $2-4$ for the Effelsberg single pulse observations and $N_{\mathrm{c}}$ range from 8 to 16 .

Given the large dimensionality of the model, we implemented the nested sampling tool POLYCHORD (Handley et al. 2015) to efficiently sample the parameter space instead of using traditional $\chi^{2}$-fitting techniques. The priors on all parameters are set to be uniform, except for $A_{i j}$ where we use log-uniform priors. We refer to $1 \sigma$ error bars as the $68.3 \%$ contours of the one-dimensional marginalized posterior distribution of each parameter. The 


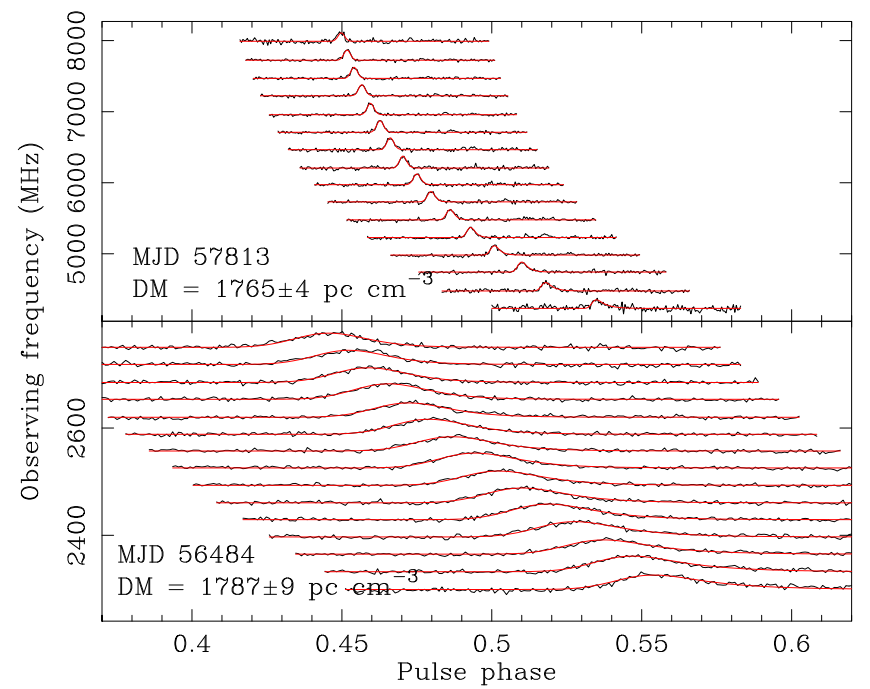

Figure 1. Scattered and dispersed observed pulse profiles of PSR J1745-2900 (over a selected phase window) are shown in black for a set of 16 subbands from a $\mathrm{C}+$ single pulse profile (top panel) and a $2.5 \mathrm{GHz}$ daily averaged profile (bottom panel). The red curves show our model of scattered and dispersed Gaussian profiles taken from the maximum likelihood solution. The MJD of the observation and the estimated DM are indicated at the bottom-left part of each panel.

resulting code can be found online. ${ }^{16}$ An example of our modeling results can be seen in Figure 1.

The DM results of the modeling of the Effelsberg and NRT data are shown in Figure 2. A linear least-squares fit to the DM values shows a marginal decrease of approximately $0.6 \%$ over four years $(2 \sigma$ consistent with no DM change $)$ with $\mathrm{DM}=1762 \pm 11 \mathrm{~cm} \mathrm{pc}^{-3}$ as measured in 2017 October. Results from the scattering measurements that come out of this joint analysis will be published elsewhere.

\section{2. $R M$ Variations}

To determine the Faraday rotation of the linearly polarized emission of the magnetar, we used the RM synthesis method (Brentjens \& de Bruyn 2005) and fitted the wrapping of the Stokes vector $Q$ and $U$ under the pulse window as previously done in Eatough et al. (2013). We employed this technique to extract the RM for all our data. In contrast to our DM results, Figure 3 shows the rapid and non-monotonic evolution of the RM during our four years of observations. Since the initial $\mathrm{RM}=-66,960 \pm 50 \mathrm{rad} \mathrm{m}^{-2}$ reported in Eatough et al. (2013), the RM changed by over 3500 units to $\mathrm{RM}=-63,402 \pm 232 \mathrm{rad} \mathrm{m}^{-2}$ in 2017 October (a relative change of $5.3 \%$ ). Our measurements are also consistent with the $\mathrm{RM}=-66,080 \pm 24 \mathrm{rad} \mathrm{m}^{-2}$ reported by Schnitzeler et al. (2016) using Australian Telescope Compact Array (ATCA) observations in 2015 May at $5.4 \mathrm{GHz}$. After a steady increase in the first few months, the signed RM increased abruptly around MJD 56566 followed by another steady increase until MJD 57450. Then the signed RM diminished up to MJD 57600 before increasing over the last year of observations. A least-squares fit to the data collected over the last year shows that RM changed by about $7.4 \mathrm{rad} \mathrm{m}^{-2}$ per day. It is worth noting that two weeks of follow-up observations of the magnetar SGR 1806-20 after an outburst phase did not show evidence for RM variations (Gaensler et al. 2005).

\footnotetext{
16 https://github.com/gdesvignes/scattering
}

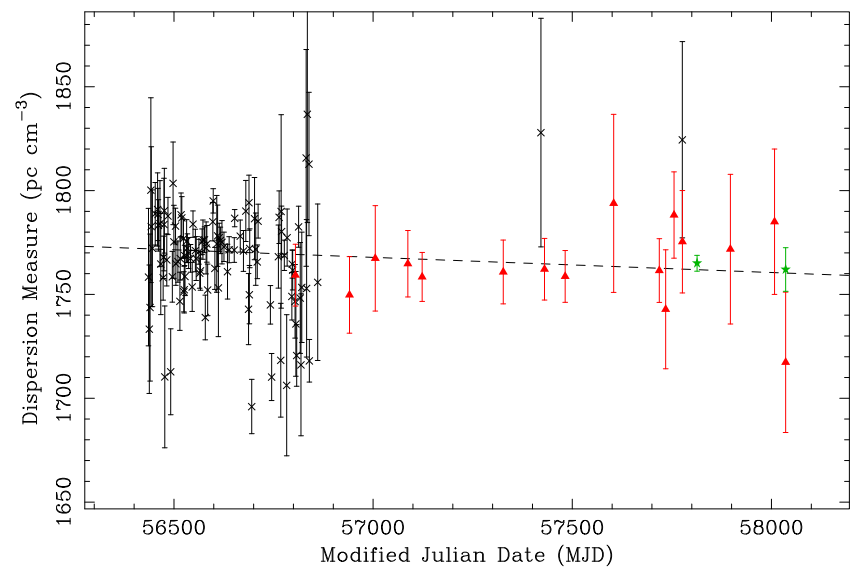

Figure 2. DM variations of the GC magnetar. The black crosses, red triangles, and green stars show the DM values (with $1 \sigma$ error bars) from the modeling of the scattered profiles from the $2.5,4.85 \mathrm{GHz}$ and $\mathrm{C}+$ data, respectively. The dashed line represents the linear least-squares fit to the data. The MJD and modeled DM are reported in the bottom-left part of each panel.

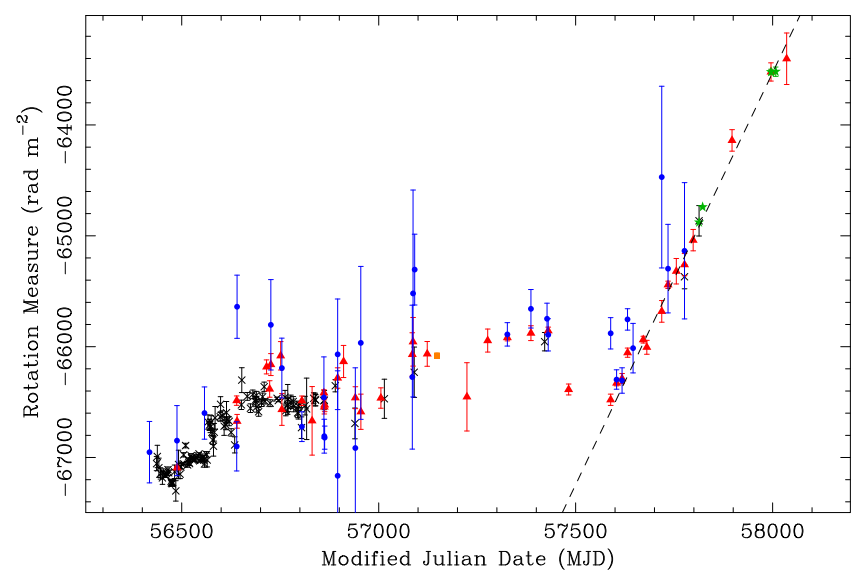

Figure 3. Variation of RM as a function of MJD. The black crosses, red triangles, blue points, and green stars show the RM values estimated from the $2.5 \mathrm{GHz}, 4.85 \mathrm{GHz}, 8.35 \mathrm{GHz}$ and $\mathrm{C}+$ data, respectively. The orange rectangle shows the value from the ATCA data recorded at $5.4 \mathrm{GHz}$ (Schnitzeler et al. 2016). The black dashed line shows the fitted slope of the recent epoch of large gradient in RM variation.

\subsection{Polarization Fraction}

Figure 4 shows the evolution of the linear polarization fraction $L_{\mathrm{f}}$ as a function of time for all our data. These results show large variations in $L_{\mathrm{f}}$ at all frequencies, with $L_{\mathrm{f}}$ at $2.5 \mathrm{GHz}$ consistently lower than at higher frequencies, by a factor of between 2 and 10 .

\section{Discussion}

The observations presented here show a clear dichotomy between the properties of the DM and RM variations toward PSR J1745-2900; the DM varies marginally, while the RM shows very large variations. This is not entirely unexpected since it has been suggested that the RM is likely caused by local magnetic phenomena, somewhere in the last few parsecs toward the GC, whereas most of the DM is accumulated along the entire line of sight to the magnetar (Eatough et al. 2013). If one considers the strong magnetic field of $8 \mathrm{mG}$ near the Bondi-Hoyle radius, a 5\% change in the local free electron density along $0.1 \mathrm{pc}$ would lead to a large RM variation of $\sim 3500 \mathrm{rad} \mathrm{m}^{-2}$ (assuming $n_{\mathrm{e}} \sim 100 \mathrm{~cm}^{-3}$ ), but a DM change 


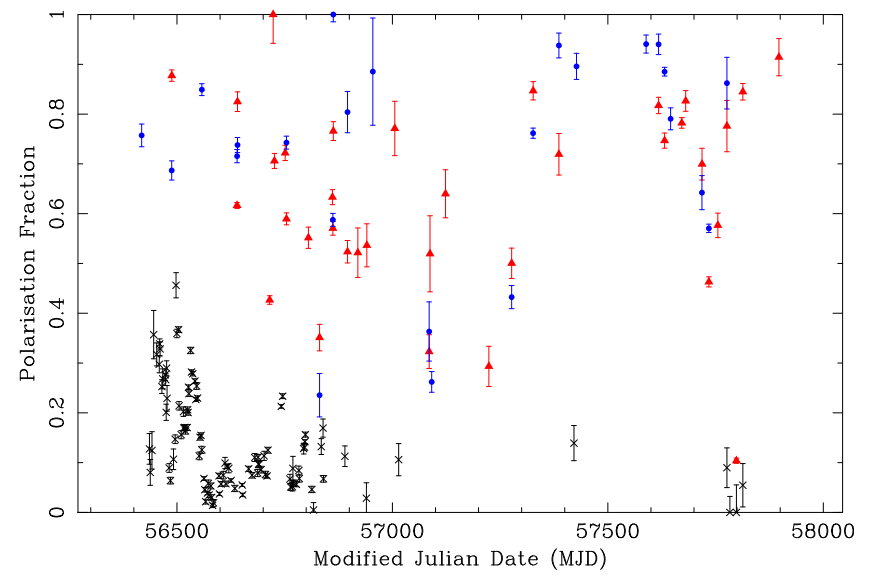

Figure 4. Polarization fraction as a function of time. The black crosses, red triangles, and blue points show the values from the $2.5 \mathrm{GHz}, 4.85 \mathrm{GHz}$, and $8.35 \mathrm{GHz}$ data, respectively. The polarization fraction at $2.5 \mathrm{GHz}$ is consistently lower than at the higher frequencies.

of only $\sim 0.5 \mathrm{pc} \mathrm{cm}^{-3}$, indistiguishable with our current data set. Therefore, our results point to magneto-ionic variations occuring in the GC.

\subsection{Structure Function Analysis}

As already mentioned, PSR J1745-2900 has a proper motion (Bower et al. 2015), so our measurements of DM and RM can be used to probe the angular and physical scales on which these fluctuations occur. For sparsely and irregularly sampled data, like these, power spectrum analyses are unsuitable. A frequently used alternative is the structure function of variations, see, e.g., Minter \& Spangler (1996). Following their notation, the ensemble average second-order structure function in DM and $\mathrm{RM}$ can be written $\mathrm{SF}_{\mathrm{DM}}=\left\langle[\mathrm{DM}(\theta)-\operatorname{DM}(\theta+\delta \theta)]^{2}\right\rangle$ and $\mathrm{SF}_{\mathrm{RM}}=\left\langle[\mathrm{RM}(\theta)-\operatorname{RM}(\theta+\delta \theta)]^{2}\right\rangle$, respectively, where $\theta$ is any given line of sight and $\delta \theta$ is the angular separation or "lag" of two measurements. In this work, the lag is given by the total magnitude of the proper motion ( $\left.6.37 \mathrm{mas} \mathrm{yr}^{-1}\right)$ multiplied by the separation in time of two DM or RM measurements. ${ }^{17}$ In addition, we corrected each pair of DM and RM used in the structure function for noise bias caused by measurement uncertainties (You et al. 2007). This is achieved by subtracting, respectively, $\left(\sigma_{\mathrm{DM}(\theta)}^{2}+\sigma_{\mathrm{DM}(\theta+\delta \theta)}^{2}\right)$ and $\left(\sigma_{\mathrm{RM}(\theta)}^{2}+\sigma_{\mathrm{RM}(\theta+\delta \theta)}^{2}\right)$, where $\sigma_{\mathrm{DM}}$ and $\sigma_{\mathrm{RM}}$ are the individual measurement errors in DM and RM. In Figure 5, we present the results from the structure function analysis. In the top panel, averaged values of $\mathrm{SF}_{\mathrm{DM}}$ are unchanging across all angular scales from $10^{-10} \mathrm{deg}$ to $10^{-5} \mathrm{deg}$. This is representative of the flat fit of the DM time series in Section 3.1. The bottom panel of Figure 5 shows $\mathrm{SF}_{\mathrm{RM}}$ over the same interval in angular scale. Unlike $\mathrm{SF}_{\mathrm{DM}}, \mathrm{SF}_{\mathrm{RM}}$ begins rising at angular scales over $10^{-8} \mathrm{deg}$, equivalent to a physical size of a few astronomical units at a GC distance of $8.3 \mathrm{kpc}$ (Gillessen et al. 2009). We define this size as the approximate smallest scale at which magneto-ionic structure in the GC is observed in our data.

The fitted slope of $\mathrm{SF}_{\mathrm{RM}}$ (black dashed line), $l=1.23 \pm 0.13$, is over $3 \sigma$ away from the expected value in the case of Kolmogorov turbulence (where $l=5 / 3$ ) assuming isotropic and

\footnotetext{
17 Here we assume that the relative proper motion between the magneto-ionic material and the magnetar is of the same order of magnitude as that between the magnetar and Sgr A*.
}

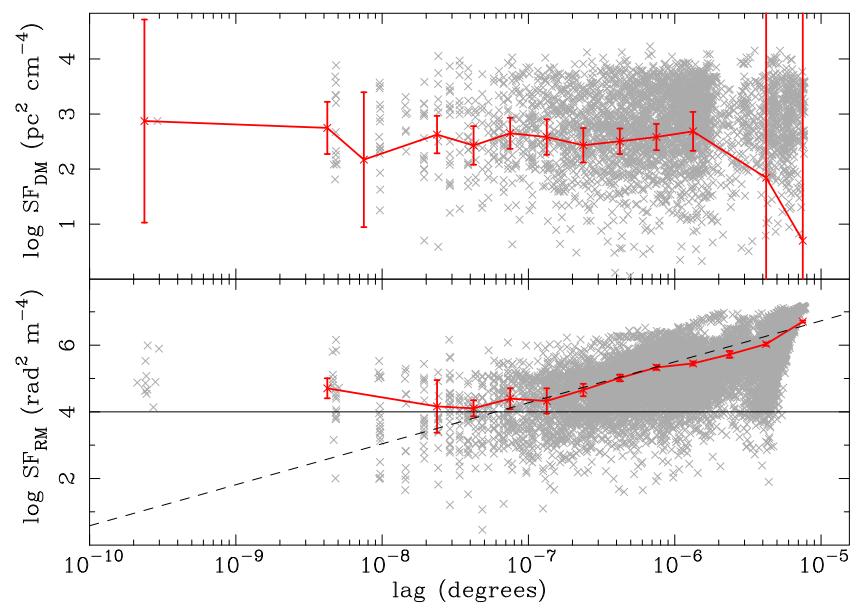

Figure 5. Structure functions, corrected for measurement errors, for the DM (top panel) and RM (bottom panel). For clarity, the error bars for each lag (gray crosses) are not shown. The red points represent the averaged values over intervals of 0.25 in log scale. The horizontal black line delimits RM variations of $100 \mathrm{rad} \mathrm{m}^{-2}$. The black dashed line shows the linear fit to the average values of $\mathrm{SF}_{\mathrm{RM}}$ allowing us to give an estimate on the angular scale at which the RM variations occur.

stationary fluctuations in RM (Simonetti et al. 1984; Lazio et al. 1990; Haverkorn et al. 2004).

\subsection{A Physical Model Based on the Depolarization}

Figure 4 shows that PSR J1745-2900 depolarizes rapidly with decreasing frequency. This could be intrinsic to the magnetar, or caused by a propagation effect. Because the former has not been observed in magnetars (e.g., Kramer et al. 2007), in this section, we model depolarization due to propagation through the interstellar medium.

For this purpose, we invoke a secondary scattering screen, close to the magnetar in the GC, in addition to the distant screen ( $\sim 6 \mathrm{kpc}$ from the GC) responsible for the temporal scatter broadening (Bower et al. 2014; Wucknitz 2014). The distant screen cannot produce the rapid RM variations we observe because of its large size $(160$ mas at $2.5 \mathrm{GHz})$ and the fact that spiral arms do not host strong magnetic fields (Haverkorn 2015). Also the size of the distant screen, when scaled to the location of the magnetar, gives a physical scale of $\sim 0.01 \mathrm{au}$, much smaller than the required physical scale (see below). Therefore, a secondary scattering screen, close to the GC, is necessary.

Scattering screens located close to the GC $(<700 \mathrm{pc})$ have been previously indicated for other GC pulsars (Dexter et al. 2017). As the magnetar moves behind or through a magnetized, ionized gas, the variation in RM that we observe over time also imprints itself as a gradient in RM across this secondary scattering disk. Following Schnitzeler et al. (2015), we can show that RM variations of order $100 \mathrm{rad} \mathrm{m}^{-2}$ within a scattering disk (Gaussian disk with a FWHM of 0.2-0.5 mas or a uniformly lit circular disk with diameter $<1$ mas at $2.5 \mathrm{GHz}$ ) is enough to explain little or no depolarization at the highest observing frequencies, and the strong or even complete depolarization at $2.5 \mathrm{GHz}$. At this frequency an RM gradient of $100 \mathrm{rad} \mathrm{m}^{-2}$ across the scattering disk would lead to PA differences up to $\sim 80^{\circ}$, leading to strong depolarization.

By considering both measurements from the RM structure function analysis outlined in Section 4.1, and the recently 
measured large gradient in RM (the fitted slope described in Section 3.2), fluctuations of the order of $100 \mathrm{rad} \mathrm{m}^{-2}$ are readily observable in our data set and occur at angular scales, or timescales, of the order of tenths of milliarcseconds and weeks respectively.

The solid horizontal black line at $\mathrm{SF}_{\mathrm{RM}}=10,000 \mathrm{rad}^{2} \mathrm{~m}^{-4}$ in Figure 5 defines the limit, where changes in RM of $100 \mathrm{rad} \mathrm{m}^{-2}$ occur. The intercept of this line with the fitted averages of $\mathrm{SF}_{\mathrm{RM}}$ gives the approximate angular scale at which these fluctuations begin to occur; which is around $6 \times 10^{-8} \mathrm{deg}(\sim 0.22$ mas or a physical scale of $\sim 1.8$ au at a GC distance of $8.3 \mathrm{kpc}$ ). The fitted slope in RM in Figure 3 indicates a change in RM of $100 \mathrm{rad} \mathrm{m}^{-2}$ occurs on timescales of just two weeks (angular displacement of $\sim 0.24$ mas, physical scale of $\sim 2.0$ au at distance of $8.3 \mathrm{kpc})$. Much shorter-term variations $(<1 \mathrm{hr})$ have been ruled out. The average value of these two measured physical scales maps to an upper bound on the scattering disk size $b$ of $\sim 1.9$ au.

The geometric scattering time delay, $\tau$, depends upon both the magnetar's distance to the screen, $z$, and its size, $b$, through $\tau=b^{2} / 2 c z . \tau$ is bounded by the observed scattering time at $2.5 \mathrm{GHz}$, and is likely substantially shorter since most of the scattering delay occurs at the midway screen (Wucknitz 2014). Following Spitler et al. (2014), we use $\tau<0.04 \mathrm{~s}$ giving $z \gtrsim 0.1$ pc. Remarkably, this places the secondary scattering screen at least $0.1 \mathrm{pc}$ in front of PSR J1745-2900, the same distance as the projected offset from Sgr A*; therefore, placing the screen close to the Bondi-Hoyle accretion radius. The observed magneto-ionic fluctuations are thus potentially occurring within the black hole reservoir. The two main assumptions in this model are the isotropy of the RM variations, and the attribution of depolarization to propagation effects.

The large RM variations toward PSR J1745-2900, along with the observed depolarization at $2.5 \mathrm{GHz}$, therefore, suggests an additional scattering screen local to the GC and embedded in a strong ambient magnetic field $(\sim 8 \mathrm{mG}$; Eatough et al. 2013). Assuming a magnetic origin for the observed RM variations, fluctuations in this field, of $\sim 12 \mu \mathrm{G}$ starting at sizes of $\sim 2$ au and going up to $\sim 400 \mu \mathrm{G}$ on the largest measured scales of $\sim 300$ au can occur either due to changes in its strength or orientation. Small changes in the free electron content in this same field cannot be ruled out. Our data also suggest the RM fluctuations deviate from Kolmogorov turbulence.

Future observations of PSR J1745-2900 will better constrain the magnetic field structure around $\mathrm{Sgr} \mathrm{A}^{*}$ and can potentially be used to test if the accretion flow becomes magnetically dominated (Pen et al. 2003).

The authors acknowledge financial support by the European Research Council for the ERC Synergy Grant BlackHoleCam under contract no. 610058. K.J.L. received support from 973 program 2015CB857101, NSFC U15311243, XDB23010200, 11690024, and funding from Max-Planck Partner Group. This work was based on observations with the $100 \mathrm{~m}$ telescope of the Max-Planck-Institut für Radioastronomie at Effelsberg. The Nançay radio Observatory is operated by the Paris Observatory, associated to the French Centre National de la Recherche Scientifique (CNRS) and to the Université d'Orléans. The authors thank D.D. Xu, R. Beck, and M. Haverkorn for helpful discussions.

\section{ORCID iDs}

G. Desvignes (iD https://orcid.org/0000-0003-3922-4055

S. A. Mao (iD https://orcid.org/0000-0001-8906-7866

H. Falcke (ib https://orcid.org/0000-0002-2526-6724

M. Kramer (i) https://orcid.org/0000-0002-4175-2271

G. C. Bower (iD https://orcid.org/0000-0003-4056-9982

\section{References}

Beck, R., \& Wielebinski, R. 2013, in Planets, Stars and Stellar Systems, Vol. 5, ed. T. D. Oswalt \& G. Gilmore (Dordrecht: Springer), 641

Bower, G. C., Deller, A., Demorest, P., et al. 2014, ApJL, 780, L2

Bower, G. C., Deller, A., Demorest, P., et al. 2015, ApJ, 798, 120

Bower, G. C., Wright, M. C. H., Falcke, H., \& Backer, D. C. 2003, ApJ, 588,331

Brentjens, M. A., \& de Bruyn, A. G. 2005, A\&A, 441, 1217

Dexter, J., Deller, A., Bower, G. C., et al. 2017, MNRAS, 471, 3563

Eatough, R. P., Falcke, H., Karuppusamy, R., et al. 2013, Natur, 501, 391

Gaensler, B. M., Kouveliotou, C., Gelfand, J. D., et al. 2005, Natur, 434, 1104

Gillessen, S., Eisenhauer, F., Fritz, T. K., et al. 2009, ApJL, 707, L114

Handley, W. J., Hobson, M. P., \& Lasenby, A. N. 2015, MNRAS, 453, 4384

Haverkorn, M. 2015, in Magnetic Fields in Diffuse Media, Astrophysics and Space Science Library, Vol. 407, ed. A. Lazarian, E. M. de Gouveia Dal Pino, \& C. Melioli (Berlin: Springer), 483

Haverkorn, M., Gaensler, B. M., McClure-Griffiths, N. M., Dickey, J. M., \& Green, A. J. 2004, ApJ, 609, 776

Hotan, A. W., van Straten, W., \& Manchester, R. N. 2004, PASA, 21, 302

Kennea, J. A., Burrows, D. N., Kouveliotou, C., et al. 2013, ApJL, 770, L24 Kramer, M., Stappers, B. W., Jessner, A., Lyne, A. G., \& Jordan, C. A. 2007, MNRAS, 377, 107

Lazarus, P., Karuppusamy, R., Graikou, E., et al. 2016, MNRAS, 458, 868

Lazio, T. J., Spangler, S. R., \& Cordes, J. M. 1990, ApJ, 363, 515

Minter, A. H., \& Spangler, S. R. 1996, ApJ, 458, 194

Mori, K., Gotthelf, E. V., Zhang, S., et al. 2013, ApJL, 770, L23

Pang, B., Pen, U.-L., Matzner, C. D., Green, S. R., \& Liebendörfer, M. 2011, MNRAS, 415, 1228

Pen, U.-L., Matzner, C. D., \& Wong, S. 2003, ApJL, 596, L207

Schnitzeler, D. H. F. M., Banfield, J. K., \& Lee, K. J. 2015, MNRAS, 450, 3579

Schnitzeler, D. H. F. M., Eatough, R. P., Ferrière, K., et al. 2016, MNRAS, 459, 3005

Schnitzeler, D. H. F. M., \& Lee, K. J. 2015, MNRAS, 447, L26

Simonetti, J. H., Cordes, J. M., \& Spangler, S. R. 1984, ApJ, 284, 126

Spitler, L. G., Lee, K. J., Eatough, R. P., et al. 2014, ApJL, 780, L3

Torne, P., Eatough, R. P., Karuppusamy, R., et al. 2015, MNRAS, 451, L50

Wucknitz, O. 2014, in Proc. XII European VLBI Network Symp. and Users Meeting Probing Interstellar Scattering towards the Galactic Centre with Pulsar VLBI, ed. A. Tarchi, M. Giroletti, \& L. Feretti (Trieste: SISSA), 66 Yan, W. M., Manchester, R. N., Hobbs, G., et al. 2011, Ap\&SS, 335, 485 You, X. P., Hobbs, G., Coles, W. A., et al. 2007, MNRAS, 378, 493 